ARTICULO ORIGINAL

\title{
Conocimientos y actitudes sobre encefalopatía hipóxico-isquémica e hipotermia terapéutica: Resultados de una intervención educativa.
}

\author{
Knowledge and attitudes on hypoxic-ischemic encephalopathy and therapeutic hypothermia: \\ Results of an educational intervention.
}

Jandy J. Flores Rodríguez, ${ }^{1}$ Karla L. Membreño Lozano, ${ }^{1}$ Allison M. Callejas, ${ }^{2}$ Jackeline Alger. ${ }^{3}$

\begin{abstract}
${ }^{1} M D$, Residente Tercer Año, Posgrado de Pediatría, Facultad de Ciencias Médicas (FCM), Universidad Nacional Autónoma de Honduras (UNAH) ${ }^{2}$ Pediatra Neonatóloga, Servicio de Neonatología, Hospital Escuela Universitario

${ }^{3} \mathrm{MD}, \mathrm{PhD}$, Unidad de Investigación Científica, FCM UNAH
\end{abstract}

\begin{abstract}
RESUMEN. Antecedentes: La asfixia perinatal, producida al feto/neonato por hipoxia y/o hipoperfusión tisular, puede llevar a encefalopatía hipóxico-isquémica (EHI). La hipotermia terapéutica $(\mathrm{HT})$ es eficaz para tratar EHI moderada/severa. Objetivo: Determinar conocimientos y actitudes sobre EHI e HT del personal sanitario y estudiantes antes y después de una intervención educativa, Departamento de Pediatría, Hospital Escuela (HE), Tegucigalpa, 2019. Métodos: Estudio cuasi experimental. Médicos, enfermeras, estudiantes de grado y posgrado fueron invitados a participar mediante consentimiento informado. El ensayo consistió en evaluación pre-intervención (Fase 1), seguida de intervención educativa, video y documento enviados por aplicación WhatsApp, y evaluación post-intervención (Fase 2). Se registraron características sociodemográficas. Se evaluó conocimientos (Excelente, Muy bueno, Bueno, Deficiente) y actitudes (Buena, Regular, Mala, Muy mala). Se midió el cambio (Delta Porcentaje) entre proporción de participantes de acuerdo a conocimientos y actitudes pre y post-intervención. Resultados: De 181 potenciales participantes, en Fase 1 participaron $147(81.2 \%)$ y en Fase 2, 127 (70.2\%). Inicialmente, 93.2\% (137) demostró un conocimiento deficiente y actitud buena 38.0\% (56), regular $28.0 \%$ (41), mala $32.0 \%$ (47). El conocimiento bueno aumentó en $561.2 \%$ (3.1\% versus $20.5 \%$ ), conocimiento muy bueno aumentó en $185.1 \%$ (4.7\% versus $13.4 \%)$, conocimiento deficiente disminuyó en $35.9 \%$ (92.2\% versus 59.1\%). La actitud regular aumentó en $37.0 \%$ (27.0\% versus 37.0\%), actitud mala disminuyó 30.4\% (32.8\% versus $22.8 \%$ ). Discusión: La intervención educativa mejoró conocimiento y actitud en relación a HT y EHI. La HT disponible en HE puede mejorar el desenlace de EHI. Se recomienda fortalecer las medidas educativas institucionales sobre el protocolo de HT.

Palabras clave: Asfixia neonatal, Encefalopatía hipóxico-isquémica, Hipotermia inducida, Recién nacido, Unidades de Cuidados Intensivos.
\end{abstract}

\section{INTRODUCCIÓN}

La asfixia perinatal es una lesión producida al feto o neonato por la falta de oxígeno y/o perfusión tisular que puede llevar a encefalopatía hipóxico-isquémica (EHI). ${ }^{1}$ Este es un síndrome clínico de gravedad variable, con disfunción neurológica aguda y asociado a morbimortalidad y discapacidad neurológica a largo plazo, con implicaciones sociales, éticas, legales y para la salud pública. ${ }^{1-4}$ La asfixia neonatal es considerada por la Academia Americana de Pediatría como una de las frustraciones clínicas no resueltas de la medicina neonatal contemporánea. ${ }^{4}$ El evento fisiopatológico es el estrés oxidativo a nivel cerebral. Se han identificado una serie de factores de riesgo incluyendo controles prenatales deficientes, escasa disponibilidad de recursos tecnológicos y el índice cultural., ${ }^{1,2,5}$ Además, se incluyen como causas los eventos agudos capaces de causar

Recibido: 24-10-2019; Aceptado para publicación 4-12-2019

Dirección para correspondencia: Jandy Flores, Karla L. Membreño

Correo electrónico: jandy.flores@yahoo.com; kle0120905@hotmail.com

Conflictos de interés. Los autores declaran no tener conflictos de interés en relación a este artículo.

DOI: https://doi.org/10.5377/rmh.v87i2.11903 daño neurológico al feto como desprendimiento prematuro de placenta, rotura uterina, prolapso de cordón, entre otros. ${ }^{1}$

La incidencia y prevalencia de EHI es variable entre países de altos y bajos ingresos. ${ }^{6} \mathrm{La}$ Organización Mundial de la Salud (OMS) estima que cada año hay cuatro millones de recién nacidos que presentan asfixia, de los cuales $25 \%$ fallece en el periodo neonatal y $8 \%$ antes de los 5 años. ${ }^{7}$ En México la EHI representa el $7.7 \%$ de los ingresos a Unidad de Cuidados Intensivos Neonatales (UCIN). Países como España reportan una incidencia de 0.49/1000 nacidos vivos y Cuba 1.78/1000 nacidos vivos. ${ }^{4}$ En Honduras la incidencia descrita es 1.9 por cada 100 nacidos vivos. ${ }^{8}$ Según datos del Departamento de Estadística, el Hospital Escuela para los años 2015 y 2016 registró 143 y 111 neonatos egresados con diagnóstico de EHI, respectivamente. ${ }^{9}$

Hasta hace una década no se contaba con medidas terapéuticas que contribuyeran a la disminución de la morbimortalidad y discapacidad neurológica producida por EHI. Sin embargo, mediante meta-análisis se ha demostrado la eficacia y seguridad de la Hipotermia Terapéutica (HT) en la reducción de la morbimortalidad y discapacidad neurológica a largo plazo 
y disminución de las convulsiones asociadas, mediante la disminución del daño neurológico en la etapa de falla energética secundaria. ${ }^{3-7,10} \mathrm{La} \mathrm{HT}$ es una herramienta implementada como medida preventiva y terapéutica en países desarrollados y a nivel de Latinoamérica, naciones como Uruguay lo implementaron desde 2009 y Argentina desde 2011.,11

A nivel nacional, el Servicio de Recién Nacidos del Departamento de Pediatría del Hospital Escuela, inició el uso de HT en el manejo de la EHI en abril de 2017, aunque esta herramienta terapéutica ya se encontraba descrita en los Protocolos de Manejo Materno Neonatal de la Secretaria de Salud desde el año 2016. ${ }^{12}$ En el Hospital Escuela se realiza HT activa mediante enfriamiento corporal total con una temperatura objetivo de $33{ }^{\circ} \mathrm{C}$, en un proceso de tres fases (fase de enfriamiento, fase de mantenimiento y fase de recalentamiento). En la Figura 1 se presenta el equipo utilizado. Fundamentados en esta experiencia, se consideró importante y oportuno evaluar si el personal sanitario cuenta con los conocimientos y actitudes necesarios para realizar el diagnóstico oportuno de EHI y la implementación adecuada del protocolo establecido de HT. El presente estudio se realizó con el objetivo de determinar el nivel de conocimientos y actitudes sobre EHI e HT del personal sanitario y estudiantes de grado y posgrado del Departamento de Pediatría, Hospital Escuela, Tegucigalpa, antes y después de una intervención educativa, realizada en mayo de 2019, con el propósito de contribuir a la mejor implementación de esta terapia como alternativa en el manejo de EHI.

\section{MÉTODOS}

Se realizó un estudio cuasi experimental con evaluación sobre conocimientos y actitudes acerca del tema EHI e HT antes y después de una intervención educativa en el Hospital Escuela, Tegucigalpa, durante mayo 2019. Se incluyó en el estudio a personal médico especialista en pediatría y subespecialistas en neonatología y cuidados intensivos que laboran en emergencia y sala de recién nacidos, personal profesional de enfermería y auxiliares de enfermería de Unidad de Cuidados

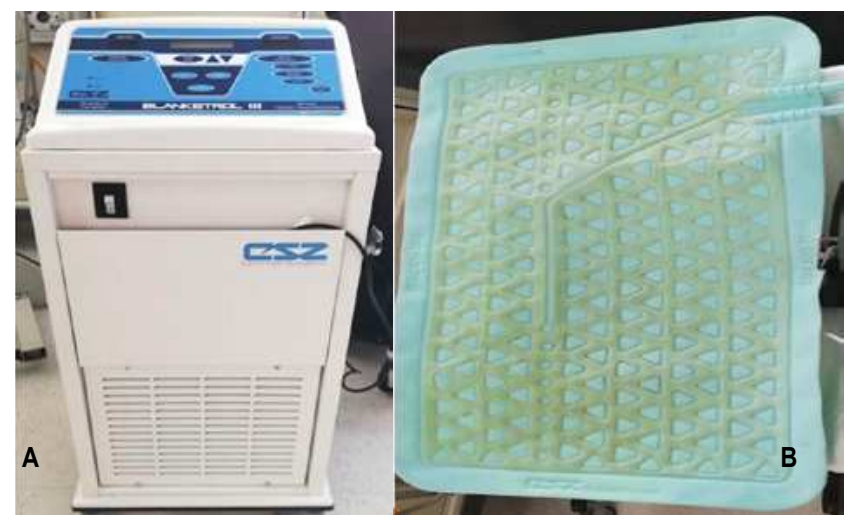

Figura 1. Máquina de hipotermia Blanketrol III, Modelo 233 (A) y accesorio manta de enfriamiento corporal total (B), Sala de Recién Nacidos, Departamento de Pediatría, Hospital Escuela, Tegucigalpa.
Intensivos Neonatales (UCIN) y Sala de Cuidados Inmediatos, médicos residentes del Posgrado de Pediatría y estudiantes del Séptimo Año de la Carrera de Medicina (estudiantes en Internado Rotatorio) de la Facultad de Ciencias Médicas UNAH que se encontraban rotando por el área de pediatría del Hospital Escuela al momento del estudio, mayo de 2019.

A partir de un total de 204 personas registradas de acuerdo a la información obtenida de la Jefatura de la Sala de Emergencia de Pediatría, Jefatura de Enfermería, Coordinación de Internado Rotatorio y Coordinación del Posgrado de Pediatría, se excluyó el personal rotando por otros hospitales, de vacaciones y con incapacidad, y se estimó una cantidad de 181 potenciales participantes. Los participantes fueron contactados en las áreas de Sala de Emergencia de Pediatría y Servicio de Recién Nacidos (Figura 2). Los criterios de inclusión fueron: personal de salud (médico especialista/sub-especialista, enfermera profesional y auxiliar de enfermería), residente de cualquier año del Posgrado de Pediatría o estudiante del Séptimo Año de la Carrera de Medicina que se encontrara rotando por el área de pediatría del Hospital Escuela, contactados en las áreas descritas, que aceptaron participar voluntariamente en el estudio y que contaran con teléfono móvil tipo smartphone. Al momento de registrarse en el estudio, a cada participante se le asignó un código.

El estudio consistió en dos fases, una evaluación pre-intervención (Fase 1), seguida de una intervención educativa y de una evaluación post-intervención (Fase 2), la cual se realizó 7 días después de la Fase 1 (Figura 2). La intervención educativa consistió en observar un video (formato mp4), ${ }^{13}$ de cinco minutos de duración complementado con la lectura de un documento como archivo PDF de dos páginas de extensión elaborados por los autores, enviados a los participantes vía electrónica utilizando la aplicación de mensajería WhatsApp. El video brindó información sobre la definición de asfixia perinatal, HT y EHI, clasificación de la EHI, criterios de inclusión y exclusión al protocolo de HT del HE, descripción de evento centinela, así como la descripción de los objetivos, complicaciones y proceso de HT. El documento en PDF presentó información detallada sobre el proceso de hipotermia terapéutica. Las instrucciones brindadas a los participantes fueron ver el video y leer el documento PDF enviados vía WhatsApp.

Se utilizaron dos instrumentos. El Instrumento 1 recolectó las características sociodemográficas de los participantes, incluyendo dos preguntas abiertas y dos cerradas. Este instrumento se aplicó en la Fase 1. El Instrumento 2 recolectó la evaluación sobre el nivel de conocimiento y tipo de actitud de los participantes y se aplicó en ambas fases, Fases 1 y 2 . La primera sección del Instrumento 2 evaluó el nivel de conocimiento mediante 13 preguntas cerradas, la cuales fueron valoradas utilizando el puntaje asignado. La segunda sección evaluó el tipo de actitud mediante 9 preguntas cerradas utilizando Escala de Likert. ${ }^{14} \mathrm{El}$ conocimiento fue evaluado como Excelente (90100 puntos), Muy bueno (80-89 puntos), Bueno (70-79 puntos) 


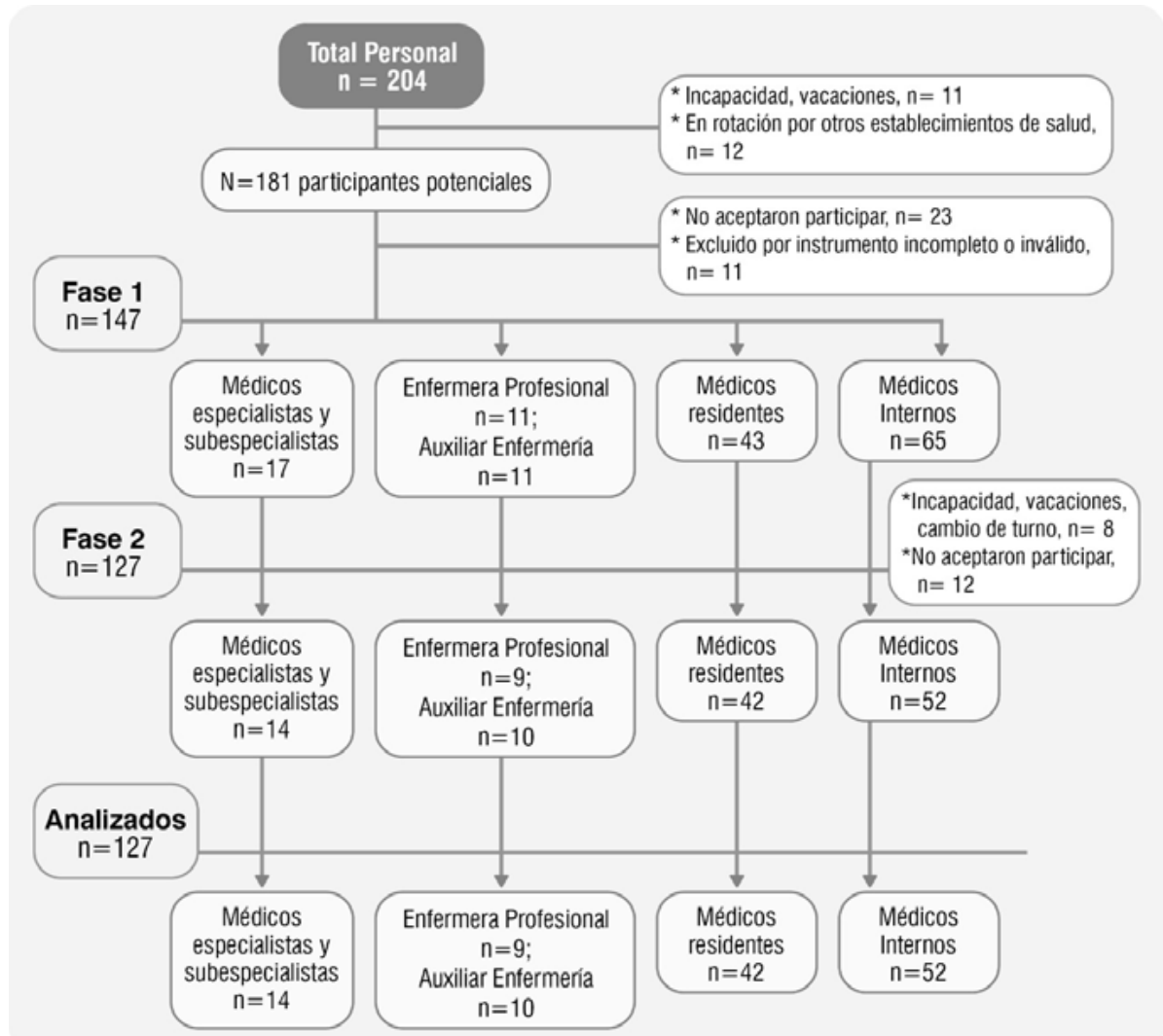

Figura 2. Diagrama de flujo de los participantes en el estudio acerca de conocimientos y actitudes sobre encefalopatía hipóxico-isquémica e hipotermia terapéutica, Hospital Escuela, Tegucigalpa, 2019.

y anónima y la información sería manejada confidencialmente, solicitando completar una encuesta auto-administrada con duración de 15-20 minutos. De igual forma se les solicitó autorización para contactarlos nuevamente para la Fase 2 del estudio. En preparación a la ejecución del estudio, los autores revisaron los aspectos éticos de la investigación mediante el curso en línea Responsabilidades de los Investigadores en cuanto a las Buenas Prácticas Clínicas, de la plataforma The Global Health Network (https://tghn.org/).

\section{RESULTADOS}

De 181 potenciales participantes, en la Fase 1 participaron 158 (87.3\%). De éstos, se excluyeron $11(7.0 \%)$ participantes, debido a respuestas incompletas 0 inválidas por lo que se contó con 147 (93.0\%) participantes para la siguiente fase. El video y documento PDF de la intervención se hicieron llegar exitosamente a los 147 (100\%) parti-

y Deficiente ( $\leq 69$ puntos). El tipo de actitud se evaluó mediante la escala de Likert, según la cual el responder totalmente de acuerdo era valorada como Buena, de acuerdo como Regular, ni de acuerdo ni en desacuerdo y en desacuerdo como Mala y totalmente en desacuerdo como Muy mala.

La información fue registrada en una base de datos del Programa Epi-info versión 7.2.2 (Centro de Control de Enfermedades, CDC, Atlanta, Georgia, EUA). Los resultados se presentan como frecuencias y porcentajes de las variables estudiadas; promedio y desviación estándar para la edad y años de experiencia laboral. Se relacionó el nivel de conocimiento de acuerdo a formación profesional y años de experiencia laboral y el tipo de actitud con el nivel de conocimiento. La medición del cambio en la proporción de participantes de acuerdo con el nivel de conocimiento y al tipo de actitud antes y después de la intervención, se realizó utilizando Delta Porcentaje $(\Delta \%)$, mediante la fórmula: $\Delta \%=$ Proporción post-intervención - Proporción preintervención / Proporción pre-intervención x 100\%.

Para la realización del estudio se contó con la aprobación del Departamento de Pediatría y el Posgrado de Pediatría, Facultad de Ciencias Médicas, UNAH. El protocolo fue revisado y aprobado por el Comité de Ética en Investigación del Hospital Escuela. Los participantes fueron invitados a participar mediante consentimiento informado escrito. A los participantes se les explicó que la participación en el estudio era voluntaria cipantes. En la Fase 2 se contó con 127 participantes representando $86.3 \%$ de los participantes que completaron la Fase 1 (147) y $70.2 \%$ del total de potenciales participantes (181). En la (Figura 2) se describen las diferentes razones para la no participación tanto en la Fase 1 como en la Fase 2.

Las características sociodemográficas, conocimiento y actitud basales, se describen en el Cuadro 1. El 52.3\% (77) de los participantes se encontraba en el grupo de edad 26-30 años y $16.3 \%$ (24) en el grupo $20-25$ años, con edad promedio y desviación estándar $30 \pm 6.9$ años, $69.3 \%$ (102) pertenecía al sexo femenino. El $73.4 \%$ (108) era estudiante, incluyendo grado $44.2 \%$ (65) y posgrado $29.2 \%$ (43). Para el personal de salud, el promedio y desviación estándar de años de experiencia laboral fue $4.5 \pm 5.3$ años, con 89 participantes $(60.5 \%)$ con un año 0 menos de experiencia laboral. Se evaluó con nivel de conocimiento deficiente $93.2 \%$ (137), bueno $2.7 \%$ (4) y muy bueno $4.1 \%$ (6). Se detectó un tipo de actitud buena $38.0 \%$ (56), regular $28.0 \%$ (41), mala $32.0 \%$ (47) y muy mala actitud $2.0 \%$ (3) (Cuadro 1).

En el Cuadro 2 se describe la distribución del nivel de conocimiento de acuerdo a formación profesional y años de experiencia laboral, encontrando que se detectó conocimiento muy bueno en 6 participantes (4\%), 5 médicos internos y 1 médico sub-especialista; 4 participantes tuvieron conocimiento bueno 
Cuadro 1. Características sociodemográficas, nivel de conocimiento y tipo de actitud, Conocimientos y actitudes sobre encefalopatía hipóxico-isquémica e hipotermia como herramienta terapéutica, Hospital Escuela, Tegucigalpa, 2019, $\mathrm{n}=147$.

\begin{tabular}{|c|c|c|}
\hline CARACTERISTICAS & $\mathbf{N}$ & $(\%)$ \\
\hline \multicolumn{3}{|l|}{ Edad (años) } \\
\hline $20-25$ & 24 & (16.3) \\
\hline $26-30$ & 77 & $(52.3)$ \\
\hline $31-40$ & 30 & $(20.4)$ \\
\hline $41-50$ & 11 & $(7.5)$ \\
\hline $51-60$ & 4 & $(2.7)$ \\
\hline$>60$ & 1 & $(0.7)$ \\
\hline Promedio (Rango) & $30(23-37)$ & \\
\hline Desviación estándar & +6.9 & \\
\hline \multicolumn{3}{|l|}{ Sexo } \\
\hline Femenino & 102 & $(69.3)$ \\
\hline Masculino & 45 & $(30.7)$ \\
\hline \multicolumn{3}{|l|}{$\begin{array}{l}\text { Formación profesional o técnica; } \\
\text { formación educativa en la Carrera de } \\
\text { Medicina }\end{array}$} \\
\hline Médicos Especialistas & 11 & $(7.4)$ \\
\hline Médicos Subespecialistas & 6 & $(4.1)$ \\
\hline Licenciada en Enfermería & 11 & $(7.5)$ \\
\hline Auxiliar de enfermería & 11 & $(7.5)$ \\
\hline Médicos residentes de primer año & 17 & $(11.6)$ \\
\hline Médicos residentes de segundo año & 11 & $(7.4)$ \\
\hline Médicos residentes de tercer año & 15 & $(10.2)$ \\
\hline Médicos internos & 65 & $(44.2)$ \\
\hline \multicolumn{3}{|l|}{ Experiencia laboral en HE (años) } \\
\hline$<1$ & 89 & $(60.5)$ \\
\hline $1.1-5$ & 31 & (21.1) \\
\hline $5.1-10$ & 13 & $(8.8)$ \\
\hline $10.1-15$ & 5 & $(3.4)$ \\
\hline $15.1-20$ & 4 & $(2.7)$ \\
\hline $20.1-25$ & 5 & $(3.4)$ \\
\hline Promedio (Rango) & $4.5(0.0-5.3)$ & -- \\
\hline Desviación estándar & +5.3 & -- \\
\hline \multicolumn{3}{|l|}{ Nivel de conocimiento } \\
\hline Excelente & 0 & $(0.0)$ \\
\hline Muy Bueno & 6 & $(4.1)$ \\
\hline Bueno & 4 & $(2.7)$ \\
\hline Deficiente & 137 & (93.2) \\
\hline \multicolumn{3}{|l|}{ Tipo de actitud } \\
\hline Buena & 56 & $(38.0)$ \\
\hline Regular & 41 & $(28.0)$ \\
\hline Mala & 47 & (32.0) \\
\hline Muy mala & 3 & $(2.0)$ \\
\hline
\end{tabular}

(2.7\%), 2 médicos sub-especialistas, 1 médico especialista y 1 médico interno; 137 participantes (93.2\%) tenían conocimiento deficiente, $100 \%$ de los médicos residentes, personal profesional de enfermería, y auxiliar de enfermería, $90.8 \%$ de los médicos internos, $90 \%$ de los médicos especialistas y $50 \%$ de los médicos sub-especialistas. En relación al tiempo de experiencia laboral, 73 participantes $(49.7 \%)$ tenían menos de un año de experiencia laboral, 61 (41.5\%) tenían entre 1 y 10 años y 13 $(0.8 \%)$ tenían más de 10 años. De los 6 participantes que tuvieron nivel de conocimiento muy bueno, $5(83.4 \%)$ tenían menos de un año de experiencia laboral y 1 (16.7\%) más de 10 años. Tres participantes con nivel de conocimiento bueno tenían entre 1.1 y 10 años de experiencia laboral. De los participantes con menos de 5 años de experiencia laboral, $76.8 \%$ (113) demostró nivel de conocimiento deficiente. En el Cuadro 3 se describe la distribución del tipo de actitud de acuerdo al nivel de conocimiento, encontrando que $100.0 \%$ (6) de los participantes con nivel de conocimiento muy bueno demostró buena actitud y $75.0 \%$ (3) de los participantes con nivel de conocimiento bueno demostró mala actitud. De los participantes con nivel de conocimiento deficiente $50(36.5 \%)$ y $47(34.3 \%)$ demostraron buena y mala actitud, respectivamente.

En el Cuadro 4 se describe el cambio en la proporción del nivel de conocimiento y tipo de actitud después de haber realizado la intervención educativa. El nivel de conocimiento excelente y muy bueno aumentó de $4.7 \%$ a $20.5 \%$ luego de la intervención. El nivel de conocimiento bueno aumentó 561.2\% después de la intervención educativa ( $3.1 \%$ versus $20.5 \%$ ), seguido del nivel de conocimiento muy bueno con un incremento $185.1 \%(4.7 \%$ versus $13.4 \%)$ y el nivel de conocimiento deficiente disminuyó $35.9 \%$ (92.2\% versus $59.1 \%$ ). El tipo de actitud regular aumentó $37.0 \%$ posterior a la intervención educativa $(27.0 \%$ versus $37.0 \%)$ y la actitud mala disminuyó $30.4 \%$ (32.8\% versus $22.8 \%)$.

\section{DISCUSIÓN}

En este estudio sobre conocimientos y actitudes acerca de EHI y HT entre personal sanitario y estudiantes de grado y posgrado rotando por áreas pediátricas del Hospital Escuela, se identificó que el nivel de conocimiento basal de los participantes fue deficiente en $93.2 \%$ (137) y el tipo de actitud fue buena/ regular en $66 \%$ (97). Estos resultados son similares a otros estudios sobre conocimientos y actitudes realizados sobre otros temas en participantes similares a nuestro estudio. En un estudio realizado en Irán que evaluaba el nivel de conocimiento de la población sobre enfermedades parasitarias se encontró que de 3,500 participantes $57.2 \%$ no sabía que la malaria es una enfermedad parasitaria entre otras preguntas sobre enfermedades parasitarias. ${ }^{15}$ Vita y colaboradores encontraron que $61.2 \%$ de los encuestados tenía conocimiento correcto sobre la técnica de lavado de manos. ${ }^{16}$ En el trabajo realizado en Colombia 
Cuadro 2. Distribución del nivel de conocimiento de acuerdo a formación profesional y años de experiencia laboral. Conocimientos y actitudes sobre encefalopatía hipóxico-isquémica e hipotermia, Hospital Escuela, Tegucigalpa, 2019, n=147.

\begin{tabular}{|c|c|c|c|c|c|c|}
\hline \multirow{3}{*}{ Características } & \multicolumn{6}{|c|}{ Nivel de Conocimiento } \\
\hline & \multicolumn{2}{|c|}{$\begin{array}{l}\text { Muy bueno } \\
\quad \mathrm{N}=6\end{array}$} & \multicolumn{2}{|c|}{$\begin{array}{c}\text { Bueno } \\
\mathrm{N}=4\end{array}$} & \multicolumn{2}{|c|}{$\begin{array}{l}\text { Deficiente } \\
\mathrm{N}=137\end{array}$} \\
\hline & $\mathrm{N}$ & $(\%)$ & $\mathbf{N}$ & $(\%)$ & $\mathbf{N}$ & $(\%)$ \\
\hline \multicolumn{7}{|l|}{ Formación Profesional } \\
\hline Médico Especialista & 0 & $(0.0)$ & 1 & $(9.1)$ & 10 & $(90.1)$ \\
\hline Médico Subespecialista & 1 & $(16.7)$ & 2 & (33.3) & 3 & $(50.0)$ \\
\hline Licenciada en enfermería & 0 & $(0.0)$ & 0 & $(0.0)$ & 11 & $(100.0)$ \\
\hline Auxiliar en enfermería & 0 & $(0.0)$ & 0 & $(0.0)$ & 11 & $(100.0)$ \\
\hline Residente de primer año & 0 & $(0.0)$ & 0 & $(0.0)$ & 17 & $(100.0)$ \\
\hline Residente de segundo año & 0 & $(0.0)$ & 0 & $(0.0)$ & 11 & $(100.0)$ \\
\hline Residente de tercer año & 0 & $(0.0)$ & 0 & $(0.0)$ & 15 & $(100.0)$ \\
\hline Médico Interno & 5 & $(7.7)$ & 1 & $(1.5)$ & 59 & $(90.8)$ \\
\hline \multicolumn{7}{|l|}{ Experiencia laboral en HE (años) } \\
\hline$<1$ & 5 & $(6.8)$ & 1 & (1.4) & 67 & (91.8) \\
\hline $1.1-5$ & 0 & $(0.0)$ & 1 & (2.1) & 46 & (97.9) \\
\hline $5.1-10$ & 0 & $(0.0)$ & 2 & (14.3) & 12 & (85.7) \\
\hline $10.1-15$ & 1 & $(16.7)$ & 0 & $(0.0)$ & 5 & (83.3) \\
\hline $15.1-20$ & 0 & $(0.0)$ & 0 & $(0.0)$ & 3 & $(100.0)$ \\
\hline $20.1-25$ & 0 & $(0.0)$ & 0 & $(0.0)$ & 4 & $(100.0)$ \\
\hline
\end{tabular}

Cuadro 3. Distribución del tipo de actitud de acuerdo al nivel de conocimiento sobre encefalopatía hipóxico-isquémica e hipotermia como herramienta terapéutica, Hospital Escuela, Tegucigalpa, 2019, n=147.

\begin{tabular}{|c|c|c|c|c|c|c|c|c|}
\hline \multirow{3}{*}{ Nivel de Conocimiento } & \multicolumn{8}{|c|}{ Tipo de Actitud } \\
\hline & \multicolumn{2}{|c|}{$\begin{array}{c}\text { Buena } \\
\mathrm{N}=56\end{array}$} & \multicolumn{2}{|c|}{$\begin{array}{c}\text { Regular } \\
\mathrm{N}=41\end{array}$} & \multicolumn{2}{|c|}{$\begin{array}{l}\text { Mala } \\
\mathrm{N}=47\end{array}$} & \multicolumn{2}{|c|}{$\begin{array}{c}\text { Muy mala } \\
\qquad \mathrm{N}=3\end{array}$} \\
\hline & $\mathrm{N}$ & $(\%)$ & $\mathrm{N}$ & $(\%)$ & N & $(\%)$ & $\mathrm{N}$ & $(\%)$ \\
\hline Muy bueno $(n=6)$ & 6 & $(100.0)$ & 0 & $(0.0)$ & 0 & 0.0 & 0 & 0.0 \\
\hline Bueno $(n=4)$ & 0 & 0.0 & 1 & $(25.0)$ & 0 & 0.0 & 3 & $(75.0)$ \\
\hline Deficiente $(n=137)$ & 50 & $(36.5)$ & 40 & $(29.2)$ & 47 & (34.3) & 0 & 0.0 \\
\hline
\end{tabular}

por Restrepo y colaboradores, en el que se valoró el nivel de conocimiento sobre limitación de esfuerzo terapéutico del personal de salud, encontraron que solo $14.6 \%$ de los participantes conocía el significado de limitación de esfuerzos terapéuticos y $62.4 \%$ del personal médico admitió tener dificultad para toma de decisiones relacionadas al tema. ${ }^{17}$ En otro estudio realizado en el personal de enfermería sobre nivel de conocimiento sobre violencia de género, $62 \%$ de los participantes obtuvo nivel de conocimiento medio. ${ }^{18}$ Cano-Contreras y colaboradores en un estudio realizado en México, en el cual se evaluó el nivel de conocimiento de los médicos generales sobre Helicobacter pylori, concluyeron que el nivel de conocimiento de los participantes fue deficiente. ${ }^{19}$

En nuestro estudio, posterior a la intervención educativa se observó mejoría del nivel de conocimiento para el nivel muy bueno y bueno con incrementos substanciales de $185.1 \%$ y $561.2 \%$, respectivamente. Asimismo, la disminución de la pro- porción de participantes con nivel de conocimiento deficiente fue de $35.9 \%$. Estos resultados son similares a lo observado en otros estudios donde las intervenciones educativas tienen un impacto favorable en la mejora del nivel de conocimiento y tipo de actitud. En un estudio que evaluó el nivel de conocimiento sobre el lavado de manos, se encontró que después de la intervención educativa el mejoramiento en el cumplimiento de lavado de manos pasó de $45.7 \%$ antes a $55.7 \%$ después de la intervención. ${ }^{16}$ Sin embargo, esto difiere de lo encontrado en un estudio sobre tuberculosis en el cual se observó que el $35.7 \%$ de los participantes no identificó factores de riesgo para tuberculosis y solo el $1.6 \%$ identificó de forma correcta el tratamiento de primera línea. ${ }^{20}$ En contraste los resultados del trabajo realizado en Colombia de Sánchez y colaboradores, sobre el tema captación de sintomáticos respiratorios, observó incremento de 8-25\% del nivel de conocimiento después de la intervención educativa. ${ }^{21}$ 
FLORES RODRÍGUEZ JJ, ET AL

Cuadro 4. Modificación del nivel de conocimiento y tipo de actitud posterior a una intervención educativa, Conocimientos y actitudes sobre encefalopatía hipóxico-isquémica e hipotermia, Hospital Escuela, Tegucigalpa, 2019, n=127.

\begin{tabular}{|c|c|c|c|}
\hline \multicolumn{4}{|c|}{ Proporcion (\%) } \\
\hline Caracteristicas & $\begin{array}{c}\text { Antes de la intervención } \\
\text { educativa }\end{array}$ & $\begin{array}{c}\text { Posterior a la intervención } \\
\text { educativa }\end{array}$ & $\begin{array}{c}\text { Delta porcentaje } \\
\qquad \%\end{array}$ \\
\hline \multicolumn{4}{|c|}{ Nivel de conocimiento } \\
\hline Excelente & 0.0 & 7.1 & NA \\
\hline Muv Bueno & 4.7 & 13.4 & 185.1 \\
\hline Mviuy Duefro & 3.1 & 20.5 & 561.2 \\
\hline Bueno & 92.2 & 59.1 & -35.9 \\
\hline \multicolumn{4}{|l|}{ Deficiente } \\
\hline \multicolumn{4}{|l|}{ Tipo de actitud } \\
\hline Buena & 38.5 & 38.6 & 0.26 \\
\hline Regular & 27.0 & 37.0 & 37.0 \\
\hline Negural & 32.8 & 22.8 & -30.4 \\
\hline Mala & 1.7 & 1.6 & -5.8 \\
\hline Muy mala & & & \\
\hline
\end{tabular}

Las intervenciones educativas tienen impacto positivo como intervención en enfermedades crónicas no trasmisibles como Diabetes Mellitus. Se ha descrito que después de una intervención aumentó el conocimiento sobre la enfermedad, adhesión al tratamiento medicamentoso y en las tasas de hemoglobina glicosilada de los participantes. ${ }^{22}$ De igual manera López $\mathrm{N}$ y colaboradores, encontraron que el nivel de conocimiento sobre Virus del Papiloma Humano después de una intervención educativa mejoró de un nivel de conocimiento medio (56\%) a nivel de conocimiento alto $(66 \%) \cdot{ }^{23}$ En nuestro estudio se detectó buena actitud en el $100 \%$ de los participantes con nivel de conocimiento bueno, observando una relación directamente proporcional entre el nivel de conocimiento y tipo de actitud. Esto debe alertar a las autoridades institucionales en la importancia de implementar medidas de intervención educativa tanto en el personal de salud asistencial como estudiantes y así promover mejor actitud hacia nuevas medidas terapéuticas 0 de otra índole que se implementen en la institución y esperando de esta manera contribuir a mejores prácticas. Adicionalmente, los años de experiencia laboral demostraron una relación negativa (inversamente proporcional) con el nivel de conocimiento, observando que el $100 \%$ del personal con mayor a 15 años de experiencia laboral presentaron nivel de conocimiento deficiente. Esto quizá atribuido a la falta de actualización en medidas terapéuticas novedosas en pro de los pacientes que son atendidos en la unidad.

Llama la atención que de 17 médicos especialistas/subespecialistas participantes fueron evaluados con un nivel de conocimiento deficiente $76.4 \%$ (13). Este resultado es similar a lo encontrado por Almonte y colaboradores, quienes evaluaron el nivel de conocimiento de médicos docentes encontrando conocimiento incorrecto sobre conceptos básicos de bioética. ${ }^{24} \mathrm{En}$ Colombia se evaluó el nivel de conocimiento, actitudes y prácticas de los médicos sobre medicina alternativa encontrando que
$40 \%$ de los participantes tenía estudios de posgrados y el $89 \%$ no conocía las estrategias de la OMS acerca de medicina alternativa y complementaría. ${ }^{25}$ Vaddi y colaboradores, hicieron una evaluación de pediatras sobre el nivel de conocimiento sobre espasmo de sollozo encontrando que el 95\% de los participantes respondió correctamente a la tipificación, diagnóstico y tratamiento de la enfermedad. ${ }^{26}$

En nuestro estudio, el 100\% (43) de los médicos residentes y personal de enfermería (22) se detectaron con un nivel de conocimiento deficiente y el $90.8 \%$ (65) de los médicos internos también se detectó con nivel de conocimiento deficiente. En comparación con el estudio realizado en México, en médicos residentes de cirugía se observó que tenían nivel de conocimiento deficiente sobre bioética. ${ }^{27}$ En Perú se evaluó el nivel de conocimiento de los residentes sobre medicina basada en evidencia (MBE), encontrando que solo el 19\% pudo definir medicina basada en evidencia concluyendo que los participantes no tienen un concepto adecuado de MBE. Sin embargo, se identificó que poseen actitud favorable. ${ }^{28}$ Condor y colaboradores, en su trabajo sobre conocimientos actitudes y prácticas sobre bioseguridad en cuidados intensivos en personal médico y de enfermería de la institución, encontraron que el $63.3 \%$ del personal tenía nivel de conocimiento bueno. ${ }^{29}$

Sobre el tema de conocimientos, actitudes y prácticas acerca de EHI y HT no se encontró estudios similares, tanto en Honduras como internacionalmente. En Nicaragua se evaluó el nivel de conocimiento del personal de enfermería sobre medidas de bioseguridad en los procedimientos de hemodiálisis, encontrando que el nivel de conocimiento fue bajo ya que solo el $14.3 \%$ de los participantes conoció correctamente las medidas de bioseguridad. ${ }^{30}$ También se evaluó el nivel de conocimiento del personal médico incluyendo a médicos internos sobre infecciones intrahospitalarias encontrando conocimiento bajo ya que el $70 \%$ de los participantes respondió de forma incorrecta el 
cuestionario. ${ }^{31} \mathrm{~A}$ nivel nacional se encontró un estudio tipo CAP sobre investigación en estudiantes de medicina. ${ }^{32}$

En conclusión, posterior a una intervención educativa sobre EHI y HT en personal sanitario y estudiantes rotando por áreas pediátricas en el Hospital Escuela, se observó un incremento en conocimientos y mejoría en la actitud hacia esta patología y su terapia. Se observó que la mejoría en la proporción en el nivel de conocimiento fue directamente proporcional a la formación profesional. La intervención educativa incidió positivamente en la proporción del nivel de conocimiento y tipo de actitud, disminuyendo los participantes con nivel de conocimiento deficiente, aumentando la proporción de participantes con nivel de conocimiento bueno, muy bueno y excelente. Sin embargo, aún es alto el porcentaje de participantes con nivel de conocimiento deficiente lo cual podría ser atribuido a que no se prestó la suficiente atención a la intervención educativa o bien que requieren otro tipo de intervención con mayor exposición a los temas. Por otra parte, desconocemos el nivel de retención tanto de conocimiento como de actitud en un tiempo mayor al evaluado en este estudio que fue un tiempo corto de una semana.

La EHI es un importante problema de salud pública a nivel nacional e institucional por su alto impacto en el neurodesarrollo de los recién nacidos..$^{1-4}$ Además, implica un alto costo financiero en el seguimiento de las morbilidades asociadas a la asfixia en las siguientes etapas de la vida. A nivel mundial, la hipotermia se ha implementado como medida preventiva y terapéutica segura para mejorar las tasas de morbimortalidad de los neonatos con asfixia perinata ${ }^{3-7,10}$ y como un importante esfuerzo innovador esta terapia ha sido implementada recientemente en servicio de Recién Nacidos del Hospital Escuela. En base a los resultados obtenidos y contando con la evidencia científica que la HT es un tratamiento eficaz y seguro, recomendamos al Departamento de Pediatría y Servicio de Recién Nacidos, fortalecer las medidas educativas sobre el protocolo de HT para EHI.

\section{CONTRIBUCIONES}

JF, LM y AC concibieron el estudio. JF, LM, AC y JA participaron en el diseño del estudio. JF y LM recolectaron la información en las diferentes fases del estudio. JF, LM, AC y JA participaron en el análisis de los resultados. JF y LM lideraron la redacción del artículo. Todos los autores revisaron el articulo hasta alcanzar la versión final, la cual fue aprobada por todos.

\section{AGRADECIMIENTO}

Se agradece a la Dra. Tebni Ochoa, Docente del Departamento de Pediatría, Facultad de Ciencias Médicas, UNAH, por proporcionar el espacio y las facilidades para aplicar los intrumentos durante las fases de este estudio a los médicos internos. Se agradece al Dr. Alejandro Young, Servicio de Recien Nacidos, Departamento de Pediatria, Hospital Escuela, Tegucigalpa, la revisión crítica del manuscrito.

\section{REFERENCIAS}

1. García AAl, Martínez MB, ArnaezJ, Valverde E, Quero J. Asfixia intraparto y encefalopatía hipóxico-isquémica, Protocolos Diagnóstico Terapéuticos de la AEP: Neonatología. 2da Ed. Hospital Universitario La Paz; 2008, cap 26, pag 242-252.

2. Peláez A, Reyes WJ, Pérez R, Carmenate LR, Pérez LJ, Díaz G. Factores de riesgo de la asfixia perinatal. Mediciego 2016; 22 (4): S1.

3. Silveira F, Gesuele JP, Montes de Oca R, Vidal G, Martinez V, et al. Neuroprotección en pacientes con asfixia perinatal. Arch Pediatric Urug 2016; 87(3):221-233.

4. Blanco D, García-Alix A, Valverde E, Tenorio V, Vento M, Cabañas. Comisión de Estándares de la Sociedad Española de Neonataología. An Pediatr (Barc). 2011;75 (5):341.

5. García GG, Pérez-Saad H. Hipotermia como alternativa terapéutica. Revista 16 de Abril; 2017; 56 (264): 64-72.

6. Balzo F, Maiolo S, Papoff P, Giannini L, Moretti C, et. Al. Comparación de electroencefalograma vrs resonancia magnética como factor predictor del neurodesarrollo en neonatos con Encefalopatía hipóxico isquémica, tratados con hipotermia terapéutica. Pediatric Reports 2014; (6):55 32.

7. Lemus M, Sola A, et al. Recomendaciones terapéuticas del VII Consenso Clínico de SIBEN para la encefalopatía hipóxico-isquémica neonatal. Neoreviews.2016;17(9) 554-570.

8. Guerrero MJ, Eguigurems ZI, Molinero CM. Caracterización epidemiológica de los recién nacidos con asfixia perinatal en el Hospital Escuela. Honduras Pediátrica. 2007; (1), 10-14

9. Hospital Escuela. Departamento de Estadística. Hospital Escuela, Tegucigalpa, Honduras, 2018.

10. Ghosh S, Tran L, Shuster JJ, Zupanc ML. Hipotermia terapéutica para encefalopatía hipóxico-isquémica y reducción de las convulsiones neonatales después del egreso de la Unidad de Cuidados Intensivos Neonatales. Childs Nerv Syst, 2016; DOI 10.1007/s00381-016-3321.
11. Valera M, Berazateguia JP, Saaa G, Herrera CO, SepúlvedaT, Et. Al. Implementación de un programa para prevenir el daño neurológico de la encefalopatía hipóxico-isquémica en Buenos Aires. Hipotermia terapéutica, Arch Argent Pediatr 2015; (5) 113,433-442.

12. Secretaria de Salud de Honduras. Protocolos para la atención durante la preconcepción, el embarazo, el parto, el puerperio y el neonato. Secretaría de Salud de Honduras, Tegucigalpa, Honduras, 2016.

13. Salavert AC. Formatos de video Digital, [Internet] disponible en: https:// www.monografias.com/trabajos-pdf4/formatos-video-digital/formatos-video-digital.pdf

14. Matas A. Diseño del formato de escalas tipo Likert: un estado de la cuestión. Revista Electrónica de Investigación Educativa 2018; 20 (1): 38-47.

15. Raheleh B, Molouk B, Mohammad S. Analysis of knowledge and attitudes related to parasitic infections among inhabitants of Ahvaz County, Khuzes$\tan$ Province, Iran. Acta Tropica 2019; 193: 211-216.

16. De Vita V, Weisburd G, Beltramino D, Bussi E. Conocimiento actitudes y prácticas del personal de Salud relacionados con el lavado de manos clínico en una Unidad de cuidados intensivos. Rev. Méd. Rosario 2014; 80 : 105-116.

17. Restrepo D, Cossio C, Ochoa F, Jaramillo J, Bedoya J, et al. Conocimientos, actitudes y prácticas frente a la limitación de esfuerzos terapéuticos en personal de salud de un hospital universitario. Persona y Bioética 2013; 17 (2): 216-226.

18. Valdez Sánchez CA, Fernández C, Sierra A. Violencia de género: conocimientos y actitudes de las enfermeras en atención primaria. Aten Primaria 2016; 48(10): 623-631.

19. Contreras A, Rascón O, Balmori M, Gálvez S, Maza YJ, et al. El abordaje, las actitudes y el conocimiento acerca de Helicobacter pylori en médicos generales es deficiente. ¡Hay mucho que mejorar! Revista de Gastroenterología de México 2018; 83 (1):16-24. 
20. Wilches-Luna E, Nasly L, Hernández L, Hernández O, Vélez C. Conocimientos, actitudes, prácticas y educación sobre tuberculosis en estudiantes de una facultad de salud. Revista de Salud Pública 2016; 18 (1): 129 141.

21. Sánchez A, Guerrero A, Moreno L. Intervención educativa en trabajadores de la salud sobre la captación de sintomáticos respiratorios de tuberculosis. Revista Cubana de Salud Pública 2015; 41(1): 46-56.

22. Figueira A, Boas L, Coelho A, Freitas M, Pace A. Intervenciones educativas para el conocimiento de la enfermedad, adhesión al tratamiento y control de la diabetes mellitus. Rev. Latino-Am. Enfermagem 2017; 25:e2863.

23. López $\mathrm{P}$, Marcelo N, Llontop A. Influencia de una intervención educativa en el nivel de conocimiento sobre el virus papiloma humano en madres de niñas del $5^{\circ}$ y $6^{\circ}$ grado de primaria en una estatal. Universidad Peruana Cayetano Heredia, 2017.

24. Almonte F, Blanco L, Rodríguez S, De la Cruz L, Sánchez J. Conocimientos, actitudes y prácticas sobre bioética en médicos docentes de la carrera de medicina de la ciudad de Santiago de los Caballeros. Anales de Medicina PUCMM 2016; 6 (1): 32-46.

25. Gómez L. Conocimientos, actitudes y prácticas de algunos médicos colombianos acerca de la medicina alternativa y/o complementaria. Archivos de Medicina (Col) 2017; 17 (2): 326-337.

26. Vaddi V, Sahu J, Dhawan S, Suthar R, Sankhyan N. Knowledge, Attitude and Practice (KAP) Study of Pediatricians on Infantile Spasms. The Indian Journal of Pediatrics 2018; 85: 836-840.
27. Velásquez Aviña J, Pulido Cejudo A, Ruiz Suárez M, Hurtado López L. Medición del conocimiento de bioética en residentes y médicos de base de cirugía general del Hospital General de México. Cirujano General 2011; 33 (4): 248-254.

28. Canelo C, Alarcon J, Amao Ruiz E, Beteta V, Monge E. Conocimientos, actitudes y prácticas de la medicina basada en evidencias en médicos asistentes y residentes en dos hospitales de Lima-Perú. Rev Med Hered 2007; 18(2): 76-84.

29. Cóndor P, Enríquez J, Ronceros G, Tello M, Gutiérrez E. Conocimientos, actitudes y prácticas sobre bioseguridad en unidades de cuidados intensivos de dos hospitales de Lima-Perú 2008. Revista Peruana de Epidemiología 2013; 17(1): 1-5.

30. Casaya M. Conocimientos, actitudes y prácticas del personal de enfermería sobre normas de bioseguridad en los procedimientos de hemodiálisis, Hospital Militar Dr. Alejandro Dávila Bolaños, Managua, Nicaragua. Repositorio Universidad Nacional Autónoma de Nicaragua. Managua, junio 2017. Acceso octubre 2019. Disponible en http://repositorio.unan.edu. ni/7912/1/t955.pdf

31. Hernandez Faure C, Gonzalez Treasure A, Gonzalez Rodríguez I, De la Cruz Vasquez R. Conocimientos, actitudes y prácticas relacionadas con las infecciones intrahospitalarias. Revista Investigación Científica 2019; 98 (1): 17-28.

32. Izcoa A, Díaz D, Rivas K, Yanez V, Valle J. Conocimientos, actitudes y prácticas en investigación de estudiantes de la Carrera de Medicina, Tegucigalpa - UNAH, Rev. Fac. Cienc. Méd. 2016; 12 (12): 10-17.

ABSTRACT. Background: Perinatal asphyxia, caused to the fetus/ neonate by hypoxia and/or tissue hypoperfusion, can lead to hypoxic-ischemic encephalopathy (HIE). Therapeutic hypothermia $(\mathrm{TH})$ is an effective treatment for moderate/severe HIE. Objective: To determine health staff and students' knowledge and attitudes about HIE and TH before and after an educational intervention, Department of Pediatrics, Hospital Escuela (HE), Tegucigalpa, 2019. Methods: Quasi-experimental study. Physicians, nurses and pregrade and postgraduate students were invited to participate by informed consent. The trial consisted in a pre-intervention evaluation (Phase 1), followed by educational intervention, video and document sent by WhatsApp application, and post-intervention evaluation (Phase 2). Socio-demographic characteristics were recorded. Knowledge (Excellent, Very good, Good, Poor) and attitudes (Good, Fair, Bad, Very bad) were evaluated. The change (Delta Percentage) was measured between proportion of participants according to knowledge and attitudes before and after the intervention. Results: From 181 potential participants, 147 (81.2\%) participated in Phase 1 and in Phase 2, 127 (70.2\%). Initially, 93.2\% (137) showed poor knowledge; good attitude $38.0 \%$ (56), regular 28.0\% (41), bad 32.0\% (47). Good knowledge increased by $561.2 \%$ (3.1\% versus $20.5 \%)$, very good knowledge increased by $185.1 \%$ (4.7\% versus $13.4 \%)$, poor knowledge decreased by $35.9 \%$ (92.2\% versus $59.1 \%)$. The regular attitude increased by $37.0 \%(27.0 \%$ versus $37.0 \%)$, bad attitude decreased $30.4 \%$ (32.8\% versus $22.8 \%$ ). Discussion: The educational intervention improved knowledge and attitude in relation to $\mathrm{HT}$ and $\mathrm{EHI}$. The HT available in the HE can improve the outcome of EHI. It is recommended to strengthen institutional educational measures on the HT protocol.

Keywords: Asphyxia neonatorum, Hypothermia induced, Hypoxia-ischemia brain, Infant newborn, Intensive Care Units. 